\title{
Voidage distribution around bubbles in a fluidized bed: Influence on throughflow
}

\author{
J.A. Almendros-Ibáñez ${ }^{\mathrm{a}, \mathrm{b}, *}$, D. Pallarès ${ }^{\mathrm{c}}$, F. Johnsson ${ }^{\mathrm{c}}$, D. Santana ${ }^{\mathrm{d}}$ \\ a Escuela de Ingenieros Industriales, Dpto. de Mecánica Aplicada e Ingeniería de Proyectos, Castilla La Mancha University, Campus Universitario, 02071, Albacete, Spain \\ ${ }^{\mathrm{b}}$ Renewable Energy Research Institute, Avda. de la Investigación s/n , 02071, Albacete, Spain \\ c Energy Technology, Department of Energy and Environment, Chalmers University of Technology, SE 41296 Göteborg, Sweden \\ ' Carlos III University of Madrid, ISE Research Group, Thermal and Fluid Engineering Department, Avda. de la Universidad 30,28911 Leganés, Madrid, Spain
}

\begin{abstract}
A B S T R A C T
In this work, a new method for measuring void fraction distribution around endogenous bubbles in a 2D fluidized bed is presented. The technique is based on illuminating a transparent wall 2 dimensional bed with diffuse light from the rear and recording the distribution of light that penetrates the bed. The recording is made with a high speed video camera, which gives frames with grey level corresponding to the light penetration and from which the voidage distribution around the bubbles can be determined. In this way, voidage distribution in the region very close to the bubble contour $\left(r / R_{b} \leqslant 1.2\right)$ is obtained, which was not possible in previous studies due to limitations in spatial resolution. A correlation is proposed for the voidage at the contour of the bubble, with the voidage depending on the radial position and the polar angle $\varepsilon(r, \theta)$. In addition, the effect of the voidage distribution on the throughflow crossing the bubbles was studied and an increase of $20 \%$ was determined for the average bubble geometry of the more than 100 bubbles analysed.
\end{abstract}

\section{Introduction}

In most of gas fluidized beds applications, bubbles appear when the superficial gas velocity exceeds the minimum velocity required to overcome the weight of the bed. Toomey and Johnstone [1] assumed that all the air in excess of minimum fluidization velocity passes the bed in the form of gas pockets or bubbles. This assumption is known as the "two phase theory" and was adopted by Davidson [2] in his model.

Later, Jackson [3] extended Davidson's model including the momen tum equation for the particle phase and assuming that the voidage can vary around the bubble. Jackson included the voidage variation in the function $\beta(\varepsilon)$ which defines the drag force experienced by the particles. Jackson's model has no analytical solution and an iterative method is needed to solve the system of equations. Also Murray [4] developed a model similar to Jackson's, although he confined the voidage variation in a boundary layer around the bubble. Murray linearized the equations of motion and obtained an analytical solution to the problem.

In order to verify these models, Lockett and Harrison [5] developed a capacitance probe able to measure the voidage around endogenous bubbles in a 2D fluidized bed. They demonstrated that around bubbles the voidage of the emulsion phase is higher than $\varepsilon_{m f}$ and obtained qualitative agreement with Jackson's model. Stewart [6] showed that adopting an appropriated bubble velocity correlation, the experimen tal results of Lockett and Harrison [5] agree with Jackson's model.

\footnotetext{
* Corresponding author. Escuela de Ingenieros Industriales, Dpto. de Mecánica Aplicada e Ingeniería de Proyectos, Castilla La Mancha University, Campus Universitario, 02071, Albacete, Spain. Tel.: + 34 967599200x2437, +34 967599200x8214; fax: + 34967599224. E-mail address: jose.almendros@uclm.es (J.A. Almendros-Ibáñez).
}

Nguyen et al. [7] obtained similar experimental results in a "falling" two dimensional bed with an artificial bubble made with a gauze cap clamped between the front and the back walls of the bed. In this bed, the artificial bubble was fixed, while the solids moved downward with a velocity similar to the typical bubble velocities in fluidized beds. The flow of solids was controlled by a valve.

Some years after, Collins [8] derived a general expression for the experimental results of Lockett and Harrison [5] and Nguyen et al. [7] in the form

$\frac{1 \quad \varepsilon(r)}{1 \quad \varepsilon_{m f}}=\exp \left(k_{2}\left(\frac{R_{b}}{r}\right)^{3}\right)$

where $R_{b}$ is the bubble radius (assumed circular bubbles), $r$ is the distance from the center of the bubble and $k_{2}=1 / 15$ is a constant adjusted experimentally. Eq. (1) was obtained assuming radial symmetry for the bubble and for the voidage distribution $\varepsilon=\varepsilon(r)$.

Using $X$ rays in a 3D fluidized bed, Yates et al. [9] observed the voidage distribution around a stream of injected bubbles. Buyevich et al. [10] fitted the following exponential equation to the experi mental results of Yates et al. [9]

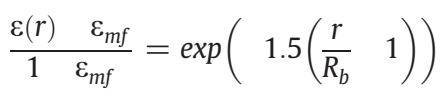

assuming again a radially symmetrical distribution.

Fig. 1 shows that Eqs. (1) and (2) give considerable difference in the voidage distribution around bubbles. The equation proposed by Buyevich et al. [10] assumed that the voidage is equal to 1 at the 


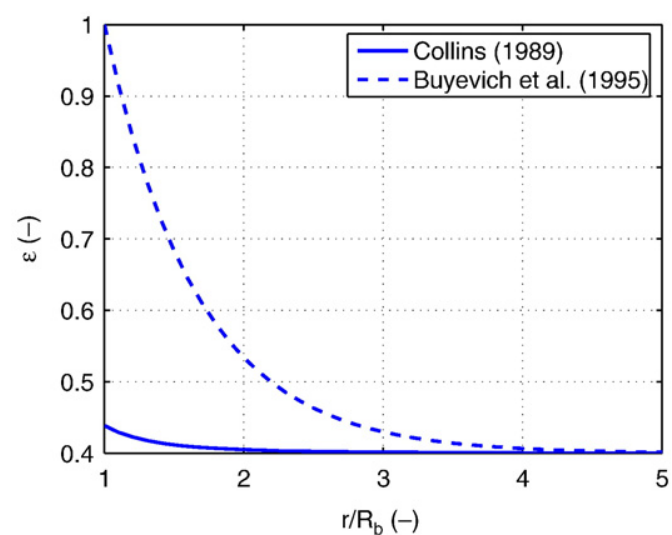

Fig. 1. Comparison of the radial voidage distribution obtained from Eqs. (1) and (2) assuming $\varepsilon_{m f}=0.4$.

bubble boundary while Collins [8] obtained a value of the voidage at the bubble contour of $\varepsilon_{b c}=0.44$, much closer to the value far away from the bubble. The observed differences can be attributed to the different bed geometries (Eq. (1) was obtained for experiments carried out in 2D fluidized beds, while Eq. (2) was obtained from 3D experiments). Also the measurement techniques employed and the uncertainty and accuracy of the measurements may have influences in the deviation between both equations.

In this work, a new method for measuring the voidage distribution around endogenous bubbles in a 2D freely bubbling fluidized bed is presented. The technique is based on illuminating the bed with a diffuse light from the rear and then measure how much light penetrates the bed. This means that light penetrates the bed when a bubble passes and no light penetrates when there is no bubble present. Around the bubble contour, there is a gradual change in light from full light to no light. The light is recorded by a high speed video camera, which then quantifies the incoming light as grey levels around the bubbles. In this way, voidage levels can be obtained in the region near the bubble contour $\left(r / R_{b} \lesssim 1.2\right)$, i.e. where Lockett and Harrison [5] and Nguyen et al. [7] could not obtain such values due to limitations in spatial resolution. A new expression for voidage distribution is proposed in the region $r / R_{b} \lesssim 1.2$.

In addition, following the work of Collins [8], the influence of the voidage variation on the throughflow crossing the bubbles was analysed numerically. For this study, the mean bubble geometry of more than 100 bubbles were analysed to obtain the voidage distribution. The numerical analysis shows that the throughflow crossing the bubble is $20 \%$ higher than the throughflow assuming a constant voidage $\varepsilon=\varepsilon_{m f}$ in the emulsion phase around the bubble.

The paper is organized as follows: first, the experimental set up and the calibration method are described. Then, the main experimen tal results, together with a correlation for the voidage distribution are presented. In the fourth section the influence of the voidage variation on the throughflow crossing the bubble are analysed numerically. Finally, the fifth section discusses the main results of the work and the sixth section summarizes the conclusions.

\section{Experimental set-up and calibration}

The experimental facility employed is similar to the one described in more detail in [11]. A 2D fluidized bed ( $1.1 \mathrm{~m}$ wide, $0.6 \mathrm{~m}$ high and $0.005 \mathrm{~m}$ thick) was used with the front and the rear walls made of glass. The rear wall of the bed was illuminated with a spotlight and onion skin paper was placed behind the bed with the aim of scattering the light as much as possible and avoiding flared highlights. A high speed video camera of 1.3 Megapixels resolution ( $1024 \times 1240$ pixels) was placed in the front of the bed. The time that the shutter was open in each picture was only $1 / 5000 \mathrm{~s}$ in order to prevent blurring by the motion of the bubbles. In all experiments the superficial velocity was varied between $1 \leq U / U_{m f} \leq 2$. Since no particle recirculation system exists in the experimental apparatus, higher fluidization velocities were not employed in order to prevent the entrainment of particles out of the bed. The height of the fixed bed during the experiments was $30 \mathrm{~cm}$ approximately achieving a resolution of $\approx 5 \mathrm{pixel} / \mathrm{mm}$.

Two types of spherical glass particles were employed in the experiments, both of them with a density of $\rho_{p}=2500 \frac{\mathrm{kg}}{\mathrm{m}^{3}}$. One group of particles had a mean diameter of $d_{p}=350 \mu \mathrm{m}$ and the other one $d_{p}=600 \mu \mathrm{m}$. Two different particle sizes were tested in order to study the influence of $d_{p}$ in the voidage distribution, although finally no noticeable differences were observed between them.

Before each experiment, two series of 100 pictures were captured for calibration. One with the empty bed and another one with the bed slightly under minimum fluidization conditions in order to avoid the presence of bubbles. The grey level obtained with the empty bed was used as a spatial calibration of voidage $\varepsilon=1$ [12]. The grey map obtained from the pictures at minimum fluidization conditions is $\varepsilon_{m f}$. In order to determine the value of $\varepsilon_{m f}$, a precisely measured mass of particles formed the bed whose free surface height at minimum fluidization conditions, $H_{m f}$, was obtained from digital image analysis. Thereby, a value of $\varepsilon_{m f}=0.4 \pm 0.002$ was calculated for both particles sizes. The uncertainty in $\varepsilon_{m f}$ was calculated assuming and uncertainty in $H_{m f}$ of $1 \mathrm{~mm}$.

The mean pictures of two calibration series are shown in Fig. 2 together with their histograms. The size of the picture captured from the central region of the bed with the camera is approximately $0.20 \times 0.25 \mathrm{~m}$. Thus, only a small section of the bed is captured in order to get a high spatial resolution. The white picture is similar to the one obtained by Goldschmidt et al. [12] who used it to correct local light intensities in their segregation study. A lighter region appears in the center of the image. During the calibration, a proper selection of the light intensity and the aperture of the diaphragm must be taken. A too high light intensity or too long times of exposure can result in the appearance of flared highlights in the center of the picture, although low intensity or short time of exposition could reduce the range of variation of the grey levels too much. In contrast, Fig. 2 shows that slightly under minimum fluidization conditions almost no light traverses the bed and the histogram is narrow and near 0 .

In literature there is a lack of studies which relate voidage to grey levels obtained with a digital camera. Two previous studies using 2D beds can be found: Poletto et al. [13] obtained data from a bed fluidized with water and Boerefijn and Ghadiri [14] measured the particle concentration in a jet using backscattering. In a different work, Yates et al. [9] measured voidage profiles around bubbles in a 3D fluidized bed using $\mathrm{X}$ rays. The experimental conditions and /or the measurement techniques employed in these works are different to the ones used in this paper.

On the other hand, there is more information about the relationship between light intensity and voidage in fluidized beds using optical probes. Optical probes measure light intensity rather than grey levels. Most of the works that can be found in the literature obtain voidage (or particle concentration) data based on the backscattering principle [15 18], i.e., measuring the light reflected by the particles rather than the light transmitted. Rizzuti and Yue [19] and Yue et al. [20] studied the light transmission through a 2D fluidized bed photoreactor with different particles sizes and bed thicknesses and observed an exponential decay of the intensity of the light transmitted with the voidage in the bed, according to Eq. (3)

$I=I_{0} \cdot \exp (a \cdot(1 \quad \varepsilon))$

where $I_{0}$ is the intensity of the emitted light, $I$ is the intensity detected by the probe and $a$ is an experimental constant.

Eq. (3) has the same form as the Beer Lambert law for light transmission through a group of suspended particles [21], although it 
(a)

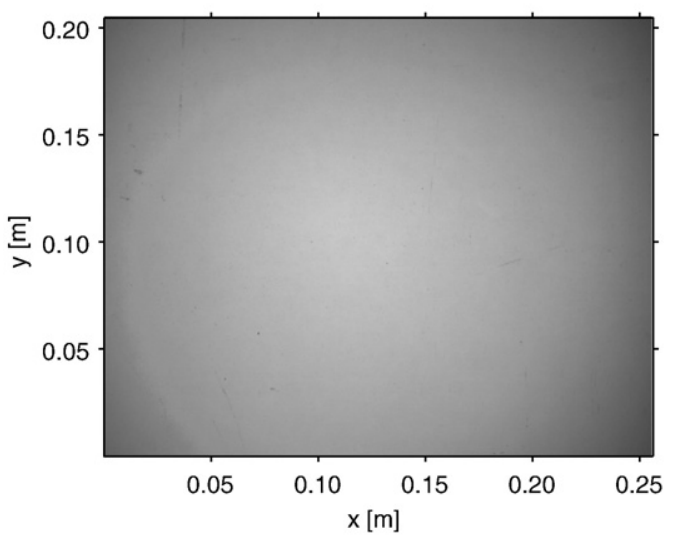

(c)

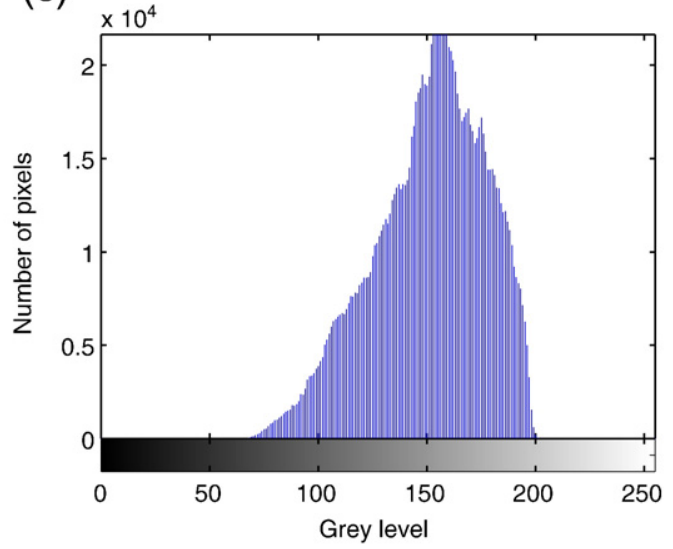

(b)

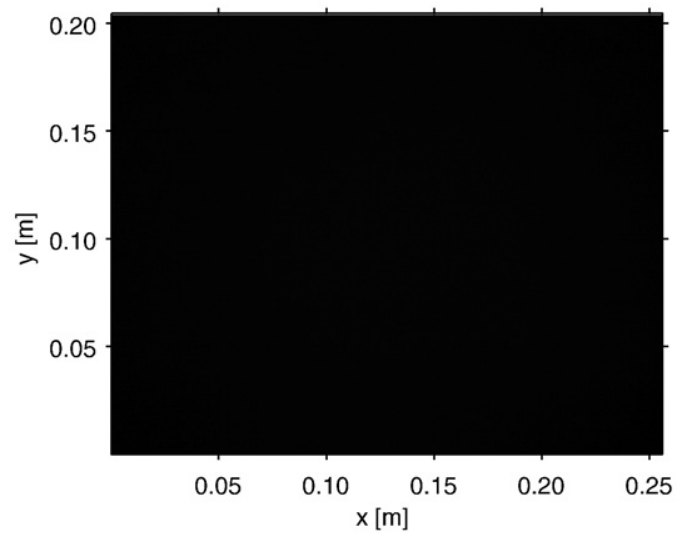

(d)

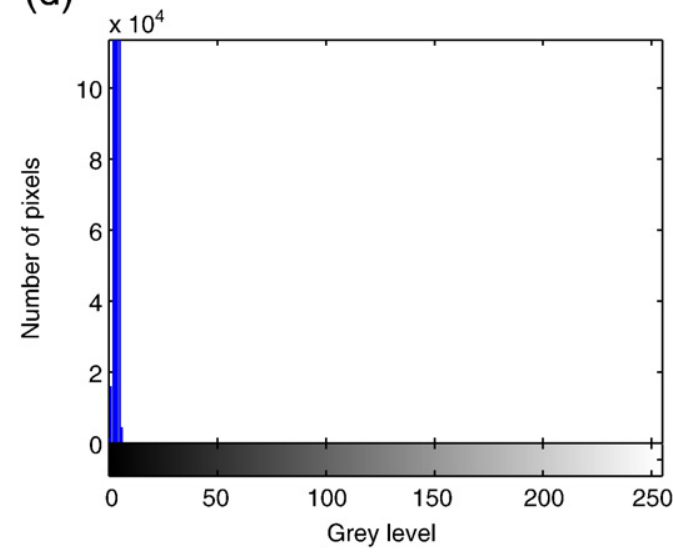

Fig. 2. (a) Average picture of the empty bed and (b) average picture with the bed at minimum fluidization condition, while (c) and (d) show the grey histograms respectively.

is important to clarify that the Beer Lambert law is not strictly applicable in the present work, because the particle concentration is high and most of the light is scattered (not absorbed). Therefore, the constant $a$ in Eq. (3) is not the absorptivity of the medium, but an empirical constant based on the previous works available in the literature using optical probes [15 20].

Nevertheless, grey levels rather than light intensities are obtained with a digital camera. According to Russ [22], there is a logarithmic relationship between the grey level (G.L.) and the light intensity:

G.L. $\propto \log \left(\frac{I}{I_{0}}\right)$

The combination of Eqs. (3) and (4) yields a linear relationship bet ween grey levels and voidage. Therefore, the two calibration pictures with the empty bed $(\varepsilon=1)$ and with the bed under minimum fluidization conditions $\left(\varepsilon=\varepsilon_{m f}=0.4\right)$ are used as spatial calibration of the framed area.

The technique employed permits to measure properly voidage values from $\varepsilon=1$ up to values close to the value at minimum fluidization conditions $(\varepsilon=0.46)$. In order obtain voidage values in the range $0.40 \lesssim \varepsilon \lesssim 0.46$, a camera with a higher grey level resolution of the one employed in this work $\left(2^{8}=256\right.$ grey levels) should be used. Neverthe less, the resolution of the camera used was found to give $2^{6}=64$ grey levels in the range of the measured voidages $(0.46 \lesssim \varepsilon \lesssim 0.70)$.

\section{Experimental results}

For each particle size, six series of pictures were taken. A total of 3272 pictures were captured at a rate of $250 \mathrm{fps}$ in each series, resulting in a total time of $13.1 \mathrm{~s}$. Approximately 10 different bubbles from each series were selected to analyse the voidage distribution. Between series, a time lag of approximately $5 \mathrm{~min}$ was needed to download the pictures from the camera to a PC. Although the camera was not recording during this time lag, the gas flow was not interrupted and the bed was in the same bubbling conditions during the six series. Therefore, the total test time for each particle size was approximately 30 min. Finally, a total of 116 different bubbles were analysed (65 bubbles from experiments with particles of $d_{p}=350 \mu \mathrm{m}$ and 51 from experiments with particles of $\left.d_{p}=600 \mu \mathrm{m}\right)$.

Fig. 3 shows a typical picture captured with the high speed video camera when the bed is freely bubbling. In the post measurement analysis a bubble was selected and then a rectangular region enclosing the bubble was cut out (Fig. 3). The bubble contour was obtained applying a local threshold value, calculated according to the method developed by Otsu [23], which gives consistent thre shold values compared with other methods [24], and is widely used due to its simplicity and stability. The method consists in separating the pixels in two classes to determine the value that maximizes the between clase variance.

The threshold value was calculated only in the neighborhood of the bubble (Fig. 3). Note that the illumination cannot be perfectly uniform (see Fig. 2) and the threshold value will be higher in the central region of the picture and lower in peripheral part of the picture. Thus, determination of a local threshold value is necessary in order to detect properly the bubble contour. Fig. 4 shows the histogram obtained for the picture shown in Fig. 3 and the threshold value that defines the bubble contour, according to the Otsu method. The histogram is wide enough and both phases (bubble and dense phase) can be distinguished properly. 


\section{(a)}

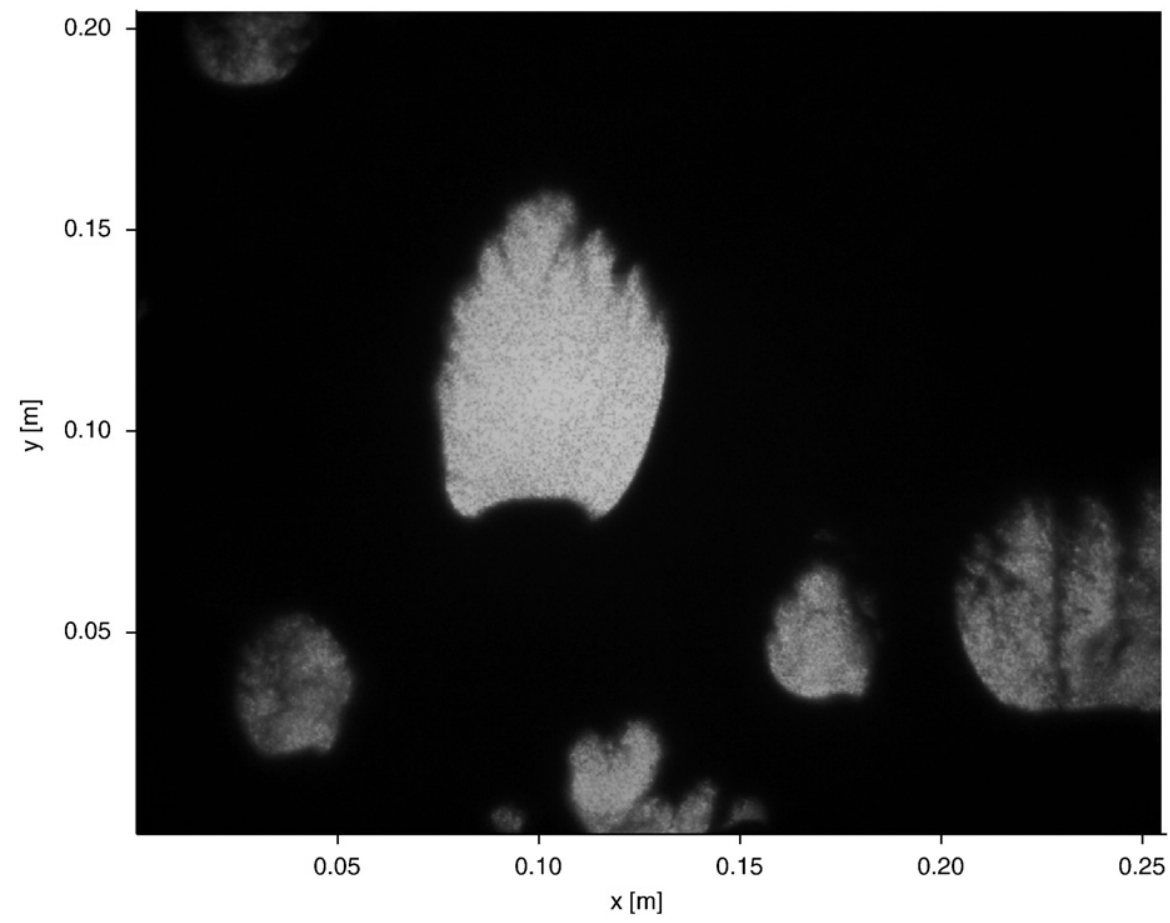

(b)

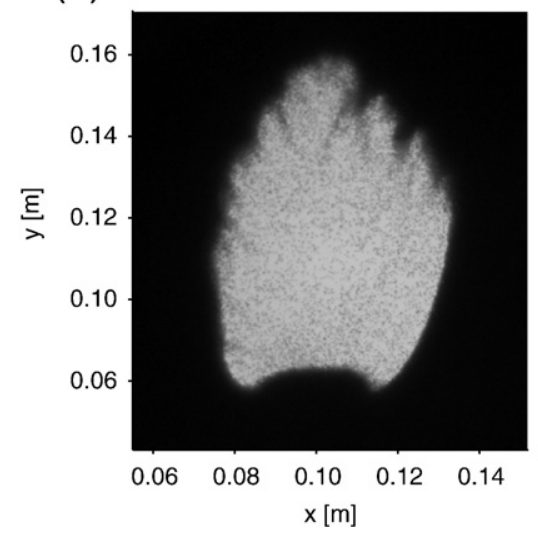

(c)

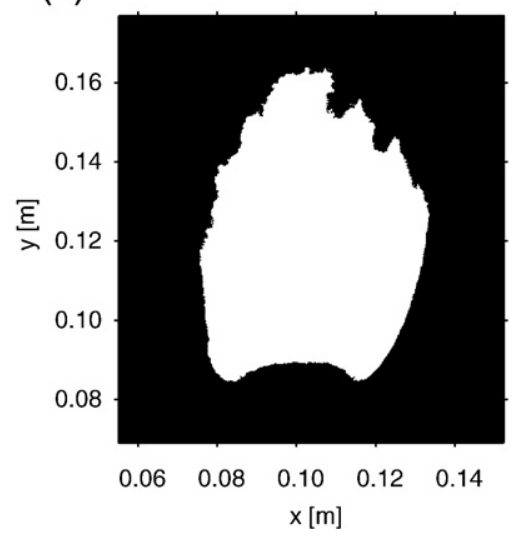

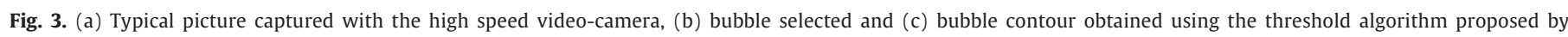
Otsu [23].

Fig. 3 shows that at the nose of the bubble typical instabilities appear and the particles rain down into the bubble in the form of "stalactites". These elongated group of particles can reach the bottom of the bubble and split the bubble in two $[25,26]$. Yet, the effect may be more common in 2D beds than in 3D beds because of wall effects.

Bubbles of different sizes and shapes were analysed. Fig. 5 shows the distribution of the bubble size, eccentricity and orientation.

The bubble size was calculated as the area equivalent diameter of the bubble ( $\sqrt{ } 4 \cdot A_{b} / \pi$ where $A_{b}$ is the projected area of the bubble). The bubble eccentricity was obtained as the eccentricity of the ellipse that has the same second moments as the bubble and the bubble orientation was defined as the angle (in degrees) between the horizontal axis and the major axis of such ellipse. The Probability Density Functions (PDF) were obtained as the distributions $p(x)$ that maximize the Shannon's entropy $\int-p(x) \ln (p(x)) d x$, restricted to a series of constrains that the distribution $p(x)$ has to satisfy, which are the raw moments of the distribution $\left\langle x^{i}\right\rangle[27,28]$ :

$\max \int-p(x) \ln (p(x)) d x$ s.t. $\int x^{i} p(x) d x=\left\langle x^{i}\right\rangle, \quad i=0, \ldots, n$

The obtained distribution is the least biased distribution that satisfies the physics embodied in the constrains. The mathematical procedure followed to obtain $p(x)$ was similar to the one described in detail in [27].

Fig. 5 shows no significant differences between the distributions of the two particle sizes. Bubbles captured from the experiments carried out with particles of $d_{p}=600 \mu \mathrm{m}$ seem to be more elongated (higher eccentricities), although the bubble size distribution is quite similar in both cases.

With the threshold value of the bubble contour, the grey level was transformed into voidage values, as is explained in Section 2. Fig. 6(a) shows the voidage at the bubble contour, $\varepsilon_{b c}$, obtained for more than 100 different bubbles. The mean value is $\bar{\varepsilon}_{b c}=0.665$. Fig. 6(b) gives the PDF of the data of $\varepsilon_{b c}$ for $d_{p}=350 \mu \mathrm{m}$ (dashed line), $d_{p}=600 \mu \mathrm{m}$ (dotted line) and both together (solid line), obtained applying again the maximum entropy method $[27,28]$. The three group of data follow a normal distribution with the data distributed around the mean value 


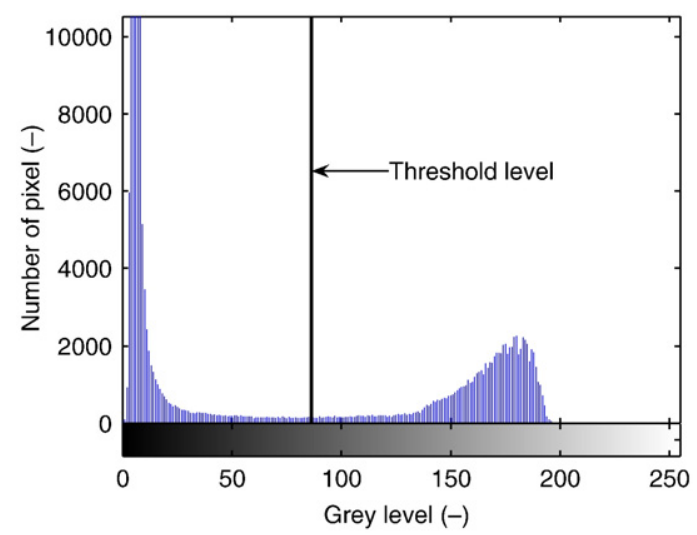

Fig. 4. Grey histogram and threshold value obtained for the picture showed in Fig. 3 (b).

$\mu$ with a standard deviation $\sigma$. Particles of higher mean diameter seem to obtain higher values of $\varepsilon_{b c}$.

Buyevich et al. [10] developed a theoretical model for the voidage distribution along the vertical axis of a circular bubble. They concluded that $\varepsilon_{b c}$ is the same at the top and at the bottom of the bubble. In addition, for a value of $\varepsilon_{m f}=0.4$, neglecting the influence of the particle pressure and applying the continuity equations across the bubble contour, they obtained values of $\varepsilon_{b c-}=0.75$ and $\varepsilon_{b c+}=0.56$ at the inside and outside bubble surface, respectively. The mean value obtained here and all individual data showed in Fig. 5 fall within these limits $\left(0.56<\varepsilon_{b c}<0.75\right)$.

Around the bubbles, different voidage contours, i.e. different grey levels, were obtained. Fig. 7 shows the results obtained for the bubble given in Fig. 3(b). The value of the voidage at the bubble contour is $\varepsilon_{b c}=0.68$. A zoom of the nose of the bubble is showed in Fig. 7(b). In the same figure the effect of the particles raining as one "stalactite" can be seen: the voidage gradient is lower in the upper region of the bubble, because the particles rain and spread in the interior of the bubble. This effect results in a more difficult detection of the bubble contour in the nose of the bubble. The voidage contours were properly detected until a minimum value of

$\varepsilon_{\min } \approx 0.46$

Lower values of voidage could not be determined because there is not enough grey range between $\varepsilon_{\min }$ and $\varepsilon_{m f}$ and also, in some cases, a closed contour of constant voidage does not appear for such low values of $\varepsilon$.

In order to obtain a voidage distribution $\varepsilon=\varepsilon(r, \theta)$ around the bubbles, 12 lines forming an angle of $\pi / 6$ rad were traced from the center of mass of each bubble. The intersection between the bubble contour and these lines defines 12 points at the bubble contour. From these starting points, 12 lines perpendicular to the contour of the bubble were traced and the intersections of these lines with the constant voidage contours defined the voidage gradient for each direction. Fig. 8 shows graphically the methodology to obtain voidage gradients for one bubble, although this procedure was repeated for all the bubbles.

Collins [8] assumed radial symmetry to adjust Eq. (1) to the experimental data of Lockett and Harrison [5] and Nguyen et al. [7]. The voidage data profiles of Lockett and Harrison [5] were obtained for bubbles with an approximately circular shape. Although some elongated bubbles were observed, only the data from the nose and from the wake were measured for these bubbles. Moreover, the geometry of the fixed artificial bubble employed by Nguyenet al. [7] was also circular. Both series of experiments resulted in voidage profiles in a region of $r / R_{b}>1.2$, where the voidage values are $\varepsilon \lesssim 0.46$. Buyevich et al. [10] also assumed radial symmetry in Eq. (2). The (a)

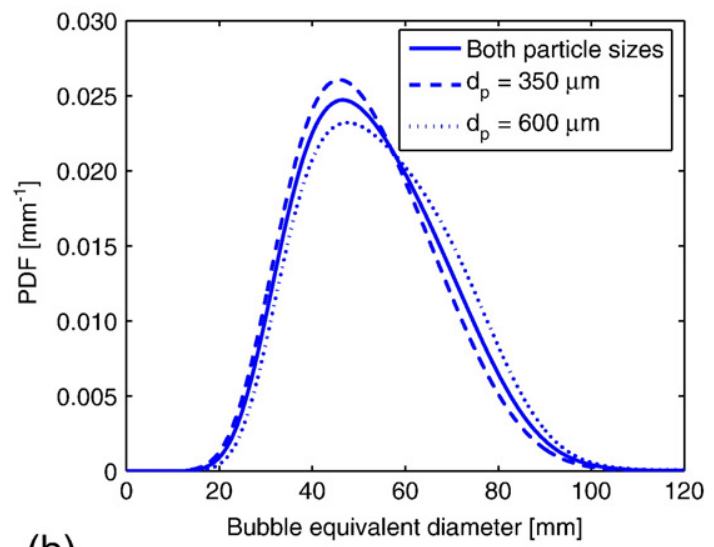

(b)

Bubble equivalent diameter [mm]

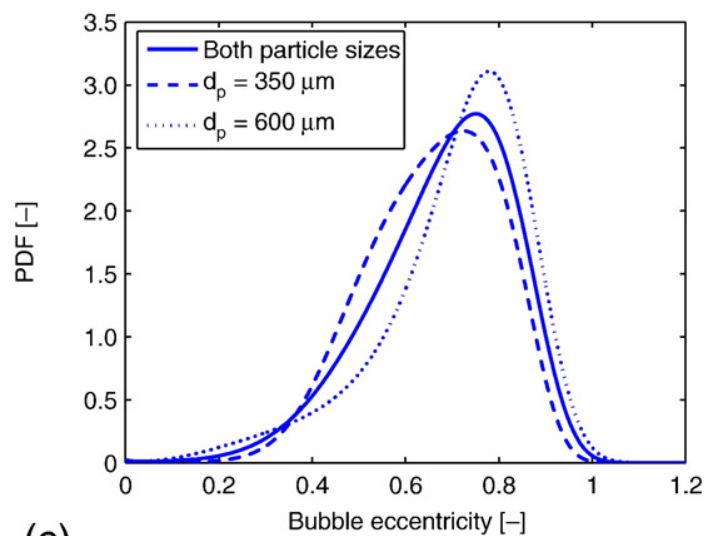

(c)

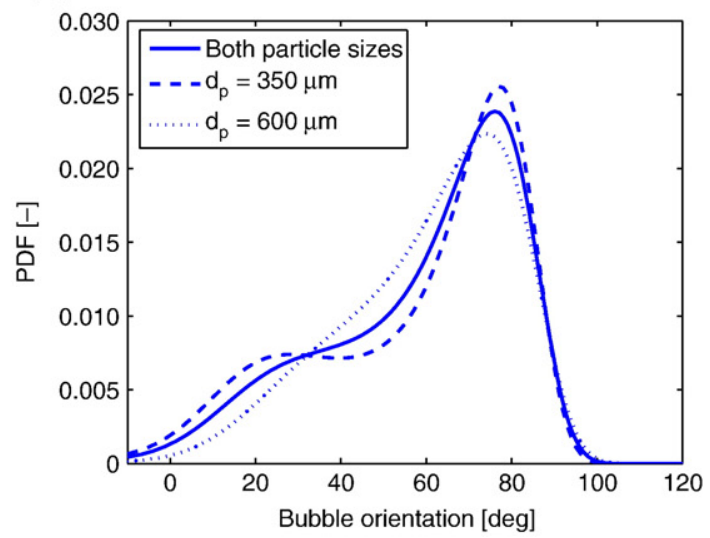

Fig. 5. Probability density functions of (a) bubble size, (b) bubble eccentricity and (c) bubble orientation. Data obtained from experiments carried out with two different particle sizes.

voidage distribution data were obtained from the horizontal section across the equator of injected bubbles and they did not observe differences along the upper half of the bubble. No data of the voidage distribution in the lower half were obtained.

In contrast, most of the bubble observed in our experiments were elongated bubbles (see Fig. 7) because of the interaction and coalescence between bubbles in freely bubbling beds. Also, lower voidage gradients in the nose of the bubble because of the effect of the "stalactites" could be expected.

In this way, $\varepsilon$ has been non dimensionalized in the same form as Buyevich et al. [10] (Eq. (2)), replacing 1 by $\varepsilon_{b c}$ due to $\varepsilon_{b c} \neq 1$ according to our experimental measurements. The non dimensional voidage $\varepsilon^{*}$ varies 
(a)

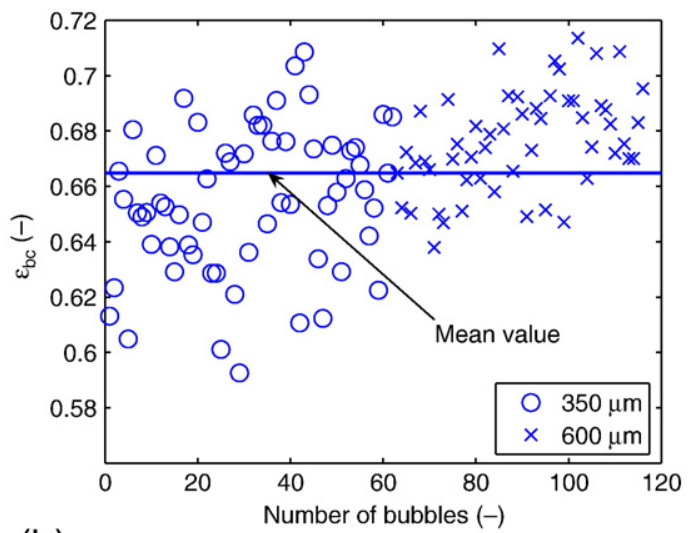

(b)

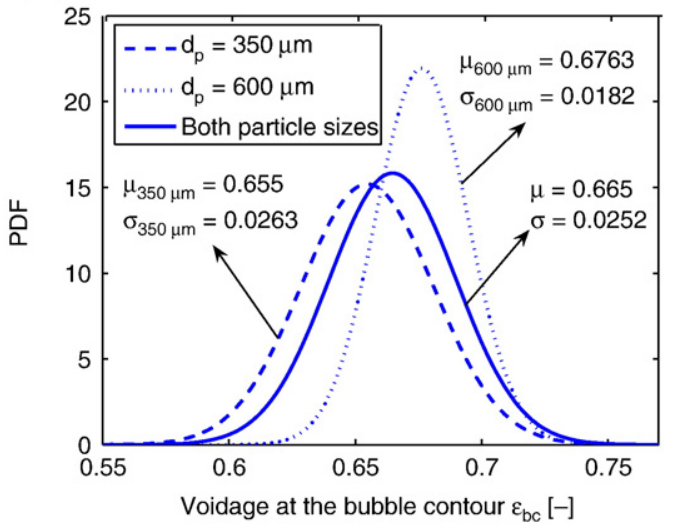

Fig. 6. (a) Voidage values at the dome contour for two different particle sizes ( $d_{p}=350 \mu \mathrm{m}$ and $d_{p}=600 \mu \mathrm{m}$ ), where the solid line indicates the mean value $\varepsilon_{b c}=0.665$ and (b) PDFs of the data showed in graph (a).

between 1 , when $\varepsilon=\varepsilon_{\max }=\varepsilon_{b c}$ and 0 when $\varepsilon=\varepsilon_{\min }=\varepsilon_{m f}$. The following equation

$\varepsilon^{*}=\frac{\varepsilon(r, \theta) \quad \varepsilon_{m f}}{\varepsilon_{b c} \quad \varepsilon_{m f}}=\exp \left(k_{1}(\theta) \cdot\left(\begin{array}{ll}\frac{r}{R_{b}} & 1\end{array}\right)\right)$

is proposed to fit the data of all bubbles in each direction, where $k_{1}(\theta)$ is a positive function of the direction. Higher values of $k_{1}(\theta)$ implies higher voidage gradients, as $\varepsilon$ decreases faster, and viceversa. The variables $\theta, R_{b}$ and $r$ are defined in Fig. 8.

In agreement with the observation of Buyevich et al. [10], no noticeable differences were observed for $k_{1}$ in the upper half of the bubble $(0 \leq \theta \leq \pi)$, although higher values of $k_{1}$ were obtained in the lower half. The voidage distribution is symmetric with respect to the vertical axis, although the value of $k_{1}$ varies with $\theta$, thus, there is not radial symmetry. Table 1 shows the numerical values of $k_{1}$ and their standard deviation for different values of $\theta$ and Fig. 9 shows graphically the variation of $k_{1}$ with $\theta$ using a cubic spline interpolation for the data between $\pi / 2 \leq \theta \leq 0$.

In the following section, the influence of the voidage distribution (Eq. (8)) on the throughflow passing the bubble is studied numerically. Higher values of voidage imply lower resistance to the gas flow. Consequently, the throughflow is higher than the one calculated assuming a constant voidage value $\varepsilon=\varepsilon_{m f}$ in the emulsion phase $[8,29]$.

\section{Influence of voidage variation on the gas flow through a bubble}

The voidage distribution around bubbles shows that, in the region in the vicinity of the dome contour $r / R_{b} \lesssim 1.2$, neither the bubble geometry nor the voidage distribution are radially symmetrical, although both of (a)

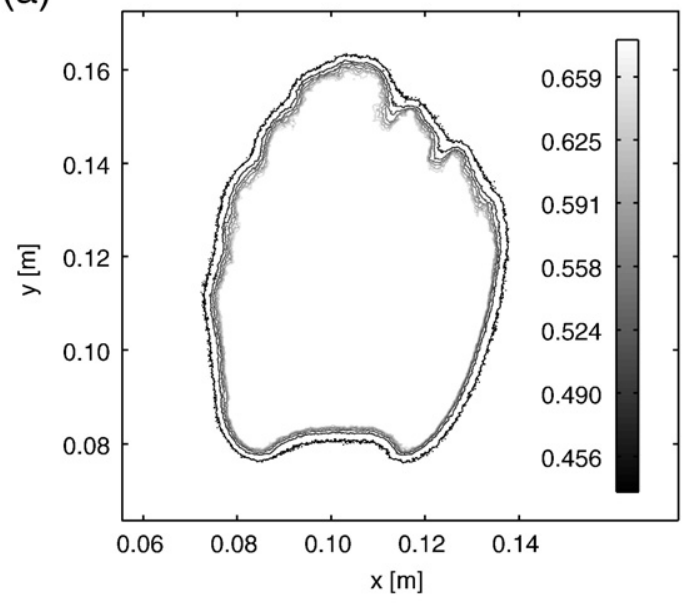

(b)

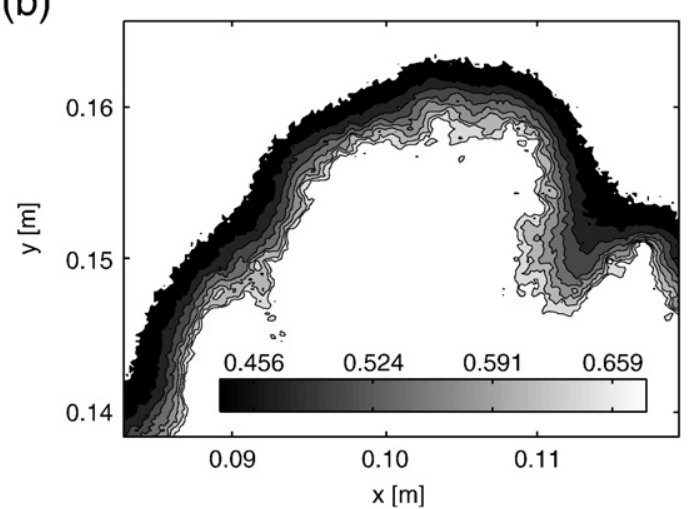

Fig. 7. (a) Voidage constant lines around the bubble showed in Fig. 3 with $\varepsilon_{b c}=0.681$ and (b) detail of the nose of the bubble with one "stalactite".

them are symmetrical with respect to the vertical axis of the bubble. Thus, in order to study the influence of the voidage variation on the gas flow around a bubble, the mean geometry of the 116 bubbles observed in the experiments is used, instead of an artificial circular or kidney shape bubble.

The mean geometry is obtained from the points detected at the bubble contour separated by $\pi / 6 \mathrm{rad}$. The mean position of each point is shown in Fig. 10 as circles and Table 2 provides the numerical values of these mean radial positions and their standard deviations for different angular positions. All the points, except the one situated at

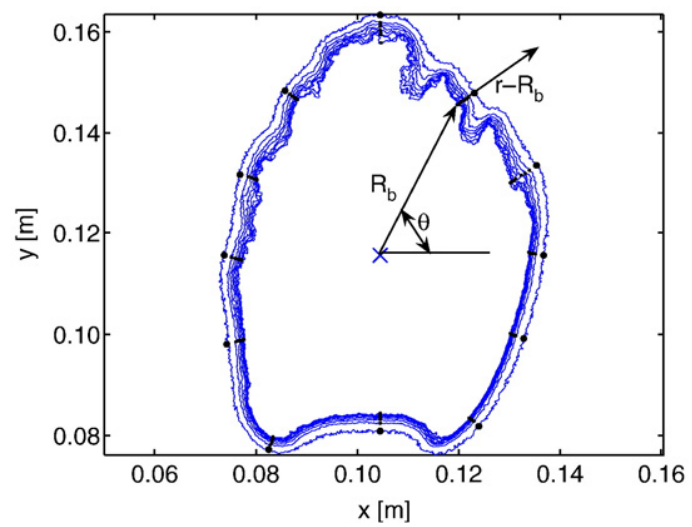

Fig. 8. Voidage variation in each direction. The points are the intersection of the constant voidage curves with the perpendicular line to the bubble contour. The cross indicates the center of mass of the bubble. 
Table 1

Experimental values of $k_{1}$ and their standard deviations for different values of $\theta$.

\begin{tabular}{cccc}
\hline$\theta$ & & $k_{1}$ & std $\left(k_{1}\right)$ \\
\hline$\left[\begin{array}{ll}0 & \pi\end{array}\right]$ & & 15.5 & 7.9 \\
$\pi / 6$, & $5 \pi / 6$ & 25.0 & 9.4 \\
$\pi / 3$, & $2 \pi / 3$ & 29.7 & 11.5 \\
$\pi / 2$ & & 22.8 & 8.8 \\
\hline
\end{tabular}

Data obtained from 116 different bubbles. Symmetry around the vertical axis is assumed.

the bubble wake $(\theta=-\pi / 2)$ fit to an ellipse of $A=31.2 \times 10^{-3} \mathrm{~m}$ and $B=23.5 \times 10^{-3} \mathrm{~m}$ using a least square technique [30], where $A$ and $B$ are the major and minor semiaxes, respectively. In contrast, the wake region of the bubble is approximately flat. Thus, the mean bubble geometry can be modeled as a truncated ellipse.

In order to study how consideration of the voidage variation around bubbles influences the flow through the bubble, numerical results obtained assuming a variable voidage outside of the bubble are compared with those obtained with constant voidage $\varepsilon=\varepsilon_{m f}$. Following the work of Collins [8] to obtain the throughflow, and assuming that the voidage inside the bubble is $\varepsilon_{b} \approx 1$, it is possible to infer that the throughflow crossing the bubble is independent of the particle movement within the bubble. Thus, to determine the throughflow crossing the bubble $U_{b}$, particle velocity is not necessary for the calculations and the equations to solve are reduced to

$\nabla \cdot(\varepsilon u)=0$

$\nabla p_{f}+\beta(\varepsilon) u=0$

Introducing the concept of the permeability of the medium, defined as $K_{e}(\varepsilon)=\mu \cdot \varepsilon / \beta(\varepsilon)$, and taking into account the relationship between the interstitial and superficial gas velocity $U=u \varepsilon$, Eqs. (9) and $(10)$ can be combined resulting in

$\nabla \cdot\left[\frac{K_{e}(\varepsilon)}{\mu} \nabla p_{f}\right]=0$

Then, the pressure field can be obtained solving Eq. (11) and the gas velocity components from Eq. (10).

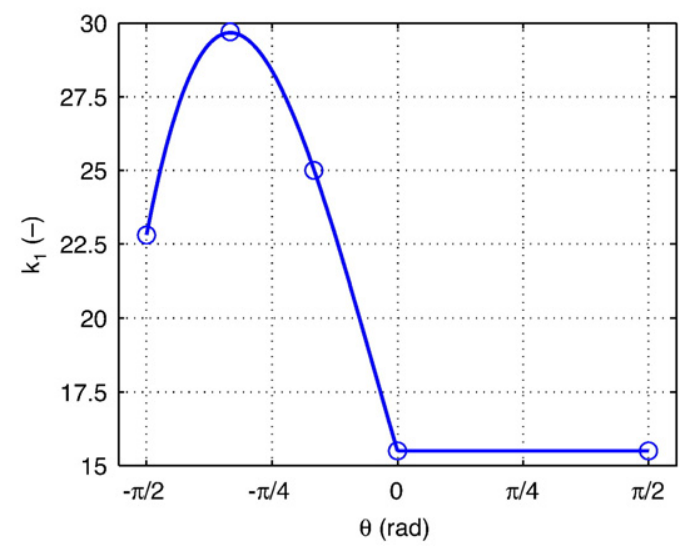

Fig. 9. Variation of $k_{1}$ with the angle $\theta$. The circles indicate the data showed in Table 1. The data are symmetric respect to the vertical axis of the bubble.

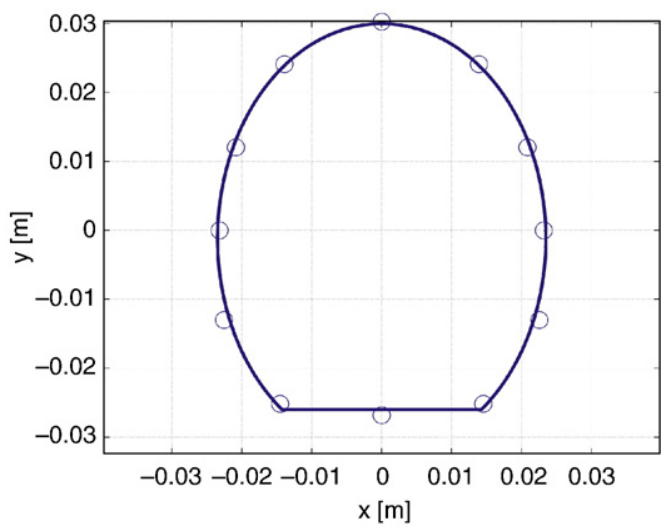

Fig. 10. Mean bubble geometry obtained from 116 bubbles.

In fact, Davidson and Harrison [31] followed the same procedure to obtain the throughflow crossing the bubble, although they assumed $\varepsilon=\varepsilon_{m f}$ and Eq. (11) is reduced to the Laplace equation

$\nabla^{2} p_{f}=0$

which was solved analytically for circular and spherical bubbles.

Different functions can be found in the literature that relate the permeability $K_{e}$ with the porosity $\varepsilon$. In this work, the linear term of Ergun equation [32], also known as the Carman Kozeny equation:

$K_{e}(\varepsilon)=\frac{\varepsilon^{3}\left(\phi d_{p}\right)^{2}}{150(1 \quad \varepsilon)^{2}}$

was employed, where $\phi$ is the particle sphericity ( $\phi=1$ in our case).

Eq. (11) was solved using a finite element program, Comsol Multi physics [33]. In order to solve numerically the problem of a bubble moving in a infinite medium, the dimensions of the domain must be significantly larger than the bubble radius, in order to avoid influences of the boundary conditions, i.e., to approximate to a bubble in a infinite suspension. Finally, a rectangular domain with dimensions of $200 \mathrm{~A}$ high and $100 B$ width was chosen, which was found to be large enough to eliminate influence of boundaries. It was found that an increase in the calculation domain does not change the final result.

Constant pressure was used as boundary condition for the bottom and pressure outlet for the top of the computational domain, because a bubble ascending in an infinite medium at minimum fluidization con ditions is assumed. The numerical value of the pressure at the bottom of the bed is not relevant because the value of the flow is imposed by the pressure drop in the bed, which is obtained by the medium permeability $K_{e}$ (Eq. (13)). No penetration of gas was chosen for the lateral boundary conditions. The same boundary conditions were assumed by Croxford [34] in order to solve the Laplace equation (Eq. (12)) to obtain the

Table 2

Mean value and standard deviation of $R_{b}$ at different angular positions for the 116 bubbles analysed.

\begin{tabular}{llc}
\hline$\theta$ & Mean $\left(R_{\mathrm{b}}\right) \times 10^{3}[\mathrm{~m}]$ & Std $\left(R_{\mathrm{b}}\right) \times 10^{3}[\mathrm{~m}]$ \\
\hline $0, \pi$ & 37.3 & 6.8 \\
$\pi / 6,5 \pi / 6$ & 34.0 & 8.2 \\
$\pi / 3,2 \pi / 3$ & 36.9 & 10.6 \\
$\pi / 2$ & 42.8 & 11.1 \\
$\pi / 6, \quad 5 \pi / 6$ & 41.2 & 8.4 \\
$\pi / 3, \quad 2 \pi / 3$ & 48.4 & 10.0 \\
$\pi / 2$ & 35.8 & 10.4
\end{tabular}

The mean values are shown graphically in Fig. 10, where a truncated ellipse is adjusted to them. 
pressure field in a freely bubbling fluidized bed and in a bed with injected bubbles. Croxford [34] compared satisfactorily the numerical results with data from experimental measurements.

The mean bubble geometry shown in Fig. 10 was situated in the middle of the domain and $\bar{\varepsilon}_{b c}=0.665$ was chosen as mean value of the voidage at the bubble contour. The voidage distribution around the bubble was calculated according to Eq. (8) using the values of $k_{1}(\theta)$ shown in Fig. 9, until the voidage reached a value of $\varepsilon=\varepsilon_{\min }=0.46$. Eq. ( 8 ) is not valid for low values of $\varepsilon$. Instead, the distribution proposed by Collins [8] (Eq. (1)), which is based on voidages $\varepsilon \leq 0.46$, obtained for $r \gtrsim 1.2$, is used. Also these data were obtained from experiments in a $2 \mathrm{D}$ bed, similar to the one used in this work. The constant $k_{2}=1 / 15$ of Eq. (1) was modified in order to get a continuous voidage distribution and avoid an abrupt change in the voidage because of the different correlations. Instead, a value of $k_{2}$ in the range [1/7 1/8] (depending on the value of $\theta$ ) was used. Fig. 11 shows the variation of $\varepsilon$ for $\theta=0$. A region very close to the bubble contour where the voidage decreases rapidly down to $\varepsilon_{\min }$ can be seen. Then the voidage decreases more slowly according to the equation proposed by Collins [8].

The permeability in the emulsion phase outside of the bubble, $k_{b}$, obtained from Eq. (13) ranged between $10^{-9}$ and $10^{-10}$ for maximum and minimum porosities respectively. The bubble interior was numer ically modeled as a region of very high permeability $K_{b} \gg K_{e}$. In this way, the bubble is a preferential path for the gas flow crossing the bed.

The numerical scheme was verified against the simplest case of an isolated circular bubble, which was analysed previously by Almendros Ibáñez et al. [35]. Having the Davidson's model as basis, Almendros Ibáñez et al. [35] obtained a flow crossing the bubble of $q_{b}=4 \cdot U_{0} \cdot R_{b}$, agreeing with the analytical result obtained by Davidson [2].

Fig. 12a and b represent, respectively, the velocity vectors $\boldsymbol{u}$ and the streamlines obtained for the mean bubble geometry viewed by a stationary observer (Bubble velocity is assumed zero in the calculations because the throughflow crossing the bubble is defined relative to the bubble [36]). The left side of each figure represents the results obtained assuming $\varepsilon=\varepsilon_{m f}=0.4$ everywhere outside the bubble and the right side the data assuming a variable voidage. Streamlines are quite similar and there is little difference between the two cases. Thus, the gas path is not affected by variations in voidage. In contrast, the moduli of the velocity vectors obtained for the constant voidage case are slightly smaller than those of the variable voidage case. An integration of the gas flow along the minor semi axis of the bubble yields

$\left.\frac{q_{b}}{U_{0} \cdot B}\right|_{\varepsilon=0.4}=4.53$

for the constant voidage case and

$\left.\frac{q_{b}}{U_{0} \cdot B}\right|_{\varepsilon=\varepsilon(r, \theta)}=5.44$

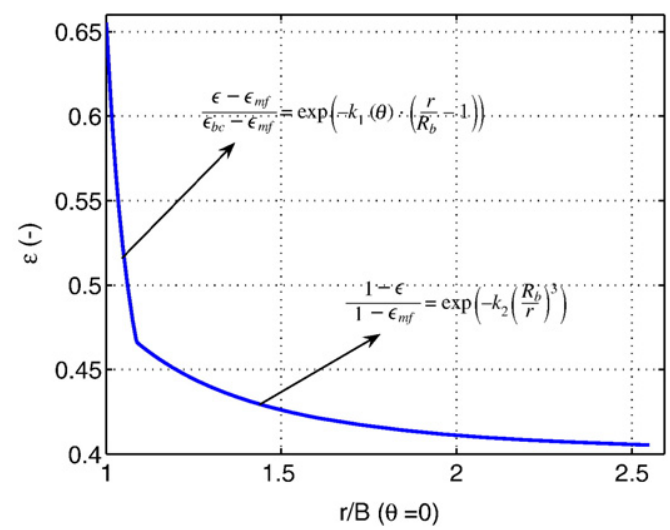

Fig. 11. Voidage distribution around the mean bubble at $\theta=0 . k_{1}=15.5$ and $k_{2}=1 / 7$. (a)

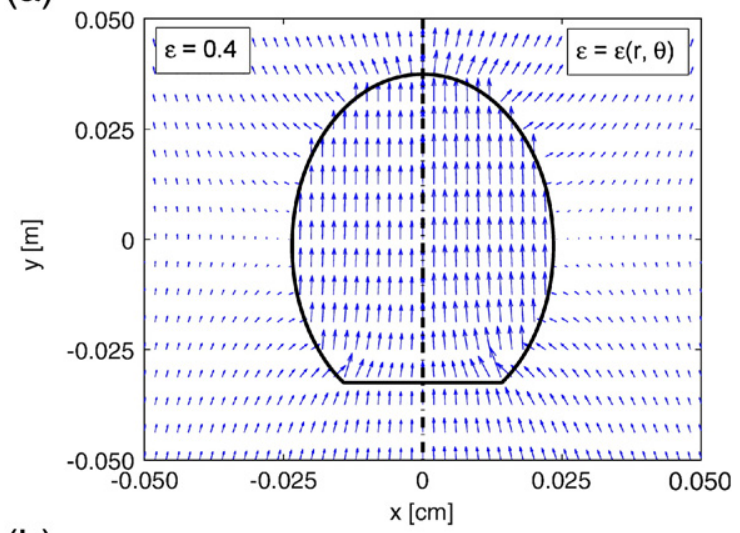

(b)

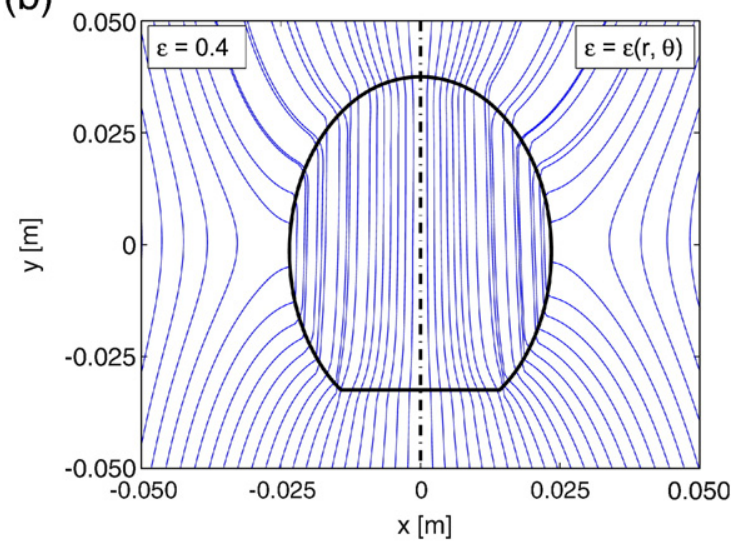

Fig. 12. Representation of $\boldsymbol{u}$, the right side of the bubble represents the result obtained with a variable voidage and the left side the result assuming a constant voidage value of $\varepsilon=\varepsilon_{m f}=0.4$ in the emulsion phase. (a) Velocity vectors and (b) Streamlines.

for the variable voidage. Thus, including the voidage distribution around the bubble results in an increases of $20 \%$ in the flow crossing the bubble. This result is logical, as higher porosities around the bubbles mean higher permeabilities and therefore lower resistance to the gas flow.

Fig. 13 shows the gas flow profiles crossing the bubble through the flat wake of the bottom of the bubble assuming variable (solid line) and constant voidage (dashed line). In both cases, in the region close to the corner $(x / D \simeq 1)$, the gas flow is much higher than in the middle region of the bubble wake $(x / D \simeq 0)$. This indicates that the truncated geometry of the bubble gives a significant increase in the flow in the region corresponding to $\theta=\pi / 3$ and $\theta=-2 \pi / 3$, where the ellipsoi dal geometry of the bubble is transformed into a flat wake.

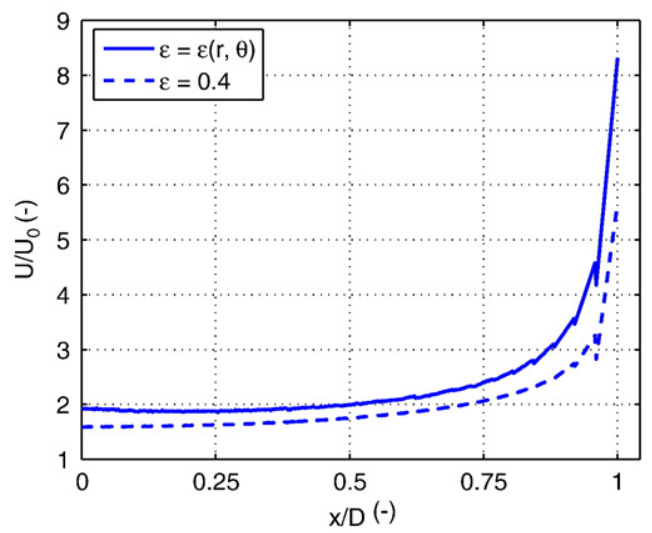

Fig. 13. Gas flow crossing the bubble through the flat region of the bubble's wake 


\section{Discussion}

Benveniste et al. [37] and Qassim et al. [38] studied the influence of the voidage variation around isolated circular bubbles, assuming a voidage distribution given by Eq. (1). They obtained an analytical solution for both, gas and particle stream functions, in the form of an infinite summation. They concluded that the voidage variation does not significantly affect the streamlines of any of the two flows (gas and solids) and the use of the Davidson's model is justified. Collins [8] following a similar reasoning developed also analytical expressions for both stream functions (although retaining only the firsts terms of the infinite sum) and justified the incompressible assumption in Davidson's model. The conclusions obtained in these previous works for circular bubbles are consistent with the results in the present work for bubbles with a truncated ellipse geometry.

In addition, Collins [8] found that the increase in the gas flow crossing a circular bubble because of the change in the voidage is only $1.5 \%$. This modest increase contrasts with the higher value of $20 \%$ obtained in this work. Nevertheless, Collins [8] assumed a voidage at the bubble contour of $\varepsilon_{b c}=0.439=1.10 \varepsilon_{m f}$, while a value of $\varepsilon_{b c}=0.665=1.66 \varepsilon_{m f}$ is experimentally determined in the present work, which lies behind the higher value of the throughflow crossing the bubble. Also Gera and Gautam [29] applied Jackson's model [3] on elliptical bubbles and observed an increase of the flow crossing the bubble with both bubble aspect ratio and voidage at the nose of the bubble.

However, not only the increase in the voidage around bubbles increases the flow crossing them. Also the geometry of the bubble plays an important role for the gas flow. The gas flow obtained from Eq. (14), i.e. 4.53, can be compared with the one obtained for a circular bubble with a radius $R_{b}=B$ [31], i.e.:

$\frac{q_{b}}{U_{0} \cdot R_{b}}=4$

or for non truncated ellipse of aspect ratio $A / B$ [39], that is,

$\frac{q_{b}}{U_{0} \cdot B}=2 \cdot\left(1+\frac{A}{B}\right)=4.66$

Thus, the gas crossing a bubble of the same cross sectional area is higher for a truncated ellipse than for the circular one, as it is expected because of the higher aspect ratio. In contrast, the throughflow is lower for a truncated elliptical bubble than for a non truncated geometry. In addition, the interface area (length, in a 2D bed) between the bubble and the dense phase seems to influence the throughflow too. A higher interface area implies a higher throughflow.

On the other hand, the voidage distribution obtained in this work is not radially symmetric, as it was assumed by Collins [8] and Buyevich et al. [10] in Eqs. (1) and (2) respectively. This fact is the result of the instabilities appearing at the nose of the bubbles, which gives the rain of particles in the form of the typical "stalactites" [26]. The value of $k_{1}(\theta)$ in Eq. (8) is higher in the bottom region of the bubble, where these instabilities do not appear. However, not only does the increase in voidage around a bubble increase the flow through them, but the geometry of the bubble also plays an important role. The maximum value of $k_{1}(\theta)$ is reached at $\theta=\pi / 3,-2 \pi / 3$ where the change in the bubble geometry occurs and also where the gas flow crossing the bubble contour is maximum.

\section{Conclusions}

In conclusion, a new method for measuring voidage distribution around bubbles in a 2D fluidized bed has been presented. This method permits to obtain voidage contours in the region very close to the bubble $\left(r / R_{b} \lesssim 1.2\right)$ where previous techniques employed by other researchers $[5,7]$ had not high enough spatial resolution. The results obtained indicate the existence of a very narrow region around the bubble where $\varepsilon$ decreases rapidly according to Eq. (8) with the values of $k_{1}(\theta)$ shown in Table 1 and Fig. 9. In addition, a mean value of $\bar{\varepsilon}_{b c}=0.665$ for the voidage at the bubble contour was obtained for a set of more than 100 bubbles obtained from experiments carried out with two different particles sizes. Finally, numerical modeling indicates that this higher voidage region surrounding the bubble results in an increase of the throughflow crossing the bubble by $20 \%$ with the representative bubble geometry of the 116 bubbles analysed.

\section{References}

[1] R.D. Toomey, H.F. Johnstone, Gaseous fluidization of solid particles, Chemical Engineering Progress 48 (1952) 220-226.

[2] J.F. Davidson, Symposium on fluidisation-discussion, Transactions of the Institution of Chemical Engineers 39 (1961) 230-232.

[3] J.R. Jackson, The mechanics of fluidised beds: Part II: The motion of fully developed bubbles, Transactions of the Institution of Chemical Engineers 41 (1963) 22-28.

[4] J.D. Murray, On the mathematics of fluidization. Part 2. Steady motion of fully developed bubbles, Journal of Fluid Mechanics 22 (1965) 57-80.

[5] M.J. Lockett, D. Harrison, The distribution of voidage fraction near bubbles rising in gasfluidized beds, in: A.A.H. Drinkenburg (Ed.), Int. Symp. on Fluidization, Netherlands University Press, Amsterdam, 1967, pp. 257-267.

[6] P.S.B. Stewart, Prediction of voidage fraction near bubbles in fluidised beds, Chemical Engineering Science 23 (1968) 396-397.

[7] X.T. Nguyen, L.S. Leung, R.H. Weiland, On void fractions around a bubble in a two dimensional fluidized bed, in: H. Angelino, J.P. Couderc, H. Gilbert, C. Lagarie (Eds.), Proc. Int. Cong. Fluidization and its Applications, Toulouse, 1973, pp. 230-239.

[8] R. Collins, A model for the effects of the voidage distribution around a fluidization bubble, Chemical Engineering Science 44 (1989) 1481-1487.

[9] J.G. Yates, D.J. Cheesman, Y.A. Sergeev, Experimental observations of voidage distribution around bubbles in a fluidized bed, Chemical Engineering Science 49 (1994) 1885-1895.

[10] Y.A. Buyevich, J.G. Yates, D.J. Cheesman, K.T. Wu, A model for the distribution of voidage around bubbles in a fluidized bed, Chemical Engineering Science 50 (1995) 3155-3162.

[11] D. Santana, S. Nauri, A. Acosta, N. García, A. Macías-Machín, Initial particle velocity spatial distribution from 2-D erupting bubbles in fluidized beds, Powder Technology 150 (2005) 1-8.

[12] M.J.V. Goldschmidt, J.M. Link, S. Mellena, J.A.M. Kuipers, Digital image analysis measurements of bed expansion and segregation dynamics in dense gas-fluidised beds, Powder Technology 138 (2003) 135-159.

[13] M. Poletto, R. Bai, D.D. Joseph, Propagation of voidage waves in a two-dimensional liquid-fluidized bed, International Journal of Multiphase Flow 21 (1995) 223-239.

[14] R. Boerefijn, M. Ghadiri, High speed video image analysis of flow of fine particles in fluidized bed jets, Advanced Powder Technology 9 (1998) 229-243.

[15] P.M. Herbert, T.A. Gauthier, C.L. Briens, M.A. Bergougnou, Application of fiber optic reflection probes to the measurement of local particle velocity and concentration in gas-solid flow, Powder Technology 80 (1994) 243-252.

[16] J.J. Nieuwland, R. Meijer, J.A.M. Kuipers, W.P.M. van Swaaij, Measurements of solid concentration and axial solids velocity in gas solid two-phase flows, Powder Technology 87 (1996) 127-139.

[17] H. Zhang, P.M. Johnston, J.X. Zhu, H.I. de Lasa, M.A. Bergougnou, A novel calibration procedure for a fiber optic solids concentration probe, Powder Technology 100 (1998) 260-272.

[18] A. Magnusson, R. Rundqvist, A.E. Almstedt, F. Johnsson, Dual fibre optical probe measurements of solids volume fraction in a circulating fluidized bed, Powder Technology 151 (2005) 19-26.

[19] L. Rizzuti, P.L. Yue, The measurement of light transmission through an irradiated fluidised bed, Chemical Engineering Science 38 (1983) 1241-1249.

[20] P.L. Yue, L. Rizzuti, V. Augugliaro, Bubble phase voidage and dense phase voidage in thin two-dimensional fluidized beds, Chemical Engineering Science 41 (1986) 171-177.

[21] A. Busciglio, G. Vella, G. Micale, L. Rizzuti, Analysis of the bubbling behaviour of 2D gas solid fluidized beds Part I. Digital image analysis technique, Chemical Engineering Journal 140 (2008) 398-413.

[22] J.C. Russ, The Image Procesing Handbook, CRC Press, 1994.

[23] N. Otsu, A threshold selection method from gray-level histograms, IEEE Transactions on Systems, Man and Cybernetics 9 (1979) 62-66.

[24] U. Gonzales-Barron, F. Butler, A comparison of seven thresholding techniques with the k-means clustering algorithm for measurement of bread-crumb features by digital image analysis, Journal of Food Engineering 74 (2006) 268-278.

[25] P.N. Rowe, B.A. Partridge, An X-ray study of bubbles in fluidised beds, Transactions of the Institution of Chemical Engineers 43 (1965) 157-175.

[26] J.A. Almendros-Ibáñez, S. Sánchez-Delgado, C. Sobrino, D. Santana, Experimental observations on the different mechanisms for solid ejection in gas-fluidized beds, Chemical Engineering and Processing 48 (2009) 734-744.

[27] D. Santana, J. Rodríguez-Rodríguez, J.A. Almendros-Ibáñez, C. Martínez-Bazán, Characteristic lengths and maximum entropy estimation from probe signals in the ellipsoidal bubble regime, International Journal of Multiphase Flow 32 (2006) 1123-1139. 
[28] C. Sobrino, J.A. Almendros-Ibáñez, D. Santana, C. Vázquez, M. de Vega, Maximum entropy estimation of the bubble size distribution in fluidized beds, Chemical Engineering Science 64 (2009) 2307-2319.

[29] D. Gera, M. Gautam, Effect of voidage variation and bubble aspect ratio on throughflow in 2-D elliptical bubbles, Powder Technology 79 (1994) 159-165.

[30] R. Halir, J. Flusser, Numerically stable direct least squares fitting of ellipses, Proc. 6 th International Conference in Central Europe on Computer Graphics and Visualization (WSCG 98), Plzen, Czech Republic, 1998, pp. 125-132.

[31] J.F. Davidson, D. Harrison, Fluidised particles, Cambridge University Press, Harrison, 1963.

[32] S. Ergun, Fluid flow through packed columns, Chemical Engineering Progress 48 (1952) 89-94.

[33] www.comsol.com (web page)

[34] A.J. Croxford, Investigation of dynamics and control of fluidised beds using pressure measurements, PhD thesis, University of Bristol (2006).

[35] J.A. Almendros-Ibáñez, D. Pallarès, F. Johnsson, D. Santana, Novel approach to characterize fluidized bed dynamics combining particle image velocimetry and finite element method, Industrial \& Engineering Chemistry Research 48 (2009) 5010-5023.

[36] D. Gera, M. Gautam, Variation of throughflow velocity in a 2-D rising bubble, Powder Technology 79 (1994) 257-263.

[37] D.E. Benveniste, S. Kinrys, R.Y. Oassim, Flow past the fluidization bubble: effect of voidage variation, in: D. Kunii, R. Toei (Eds.), Fluidization 4, Engineering Foundation, New York, 1983, pp. 9-14.

[38] R.Y. Qassim, S. Kinrys, D.E. Benveniste, Effect of voidage variation on flow past a fluidisation bubble, Chemical Engineering Science 44 (1989) 1307-1313.

[39] J.R. Grace, D. Harrison, The behaviour of freely bubbling fluidised beds, Chemical Engineering Science 24 (1969) 497-508

\section{Glossary}

$A$ : Mayor semiaxis of the ellipse $\left[31.2 \cdot 10{ }^{3} \mathrm{~m}\right]$

$A_{b}$ : Projected area of the bubble $\left[\mathrm{m}^{2}\right]$

$B$ : Minor semiaxis of the ellipse $\left[23.5 \cdot 10{ }^{3} \mathrm{~m}\right]$

$D$ : Half of the length of the bubble's flat wake $\left[16.0 \cdot 10{ }^{3} \mathrm{~m}\right]$ $d_{p}$ : Particle diameter $[\mathrm{m}]$

g: Gravity $\left[\mathrm{m} / \mathrm{s}^{2}\right]$

G.L.: Grey level [ ]

I: Light intensity [cd]

$I_{0}$ : Intensity of the incident light [cd]

$K_{e}$ : Permeability of the emulsion defined in Eq. (13) $\left[\mathrm{m}^{2}\right]$

$K_{b}$ : Permeability of the bubble interior $\left[\mathrm{m}^{2}\right]$

$k_{1}$ : Constant defined in Eq. (8) [ ]

$k_{2}$ : Constant defined in Eq. (1) [ ]

$p_{f}$ : Fluid pressure $[\mathrm{Pa}]$

$q_{b}$ : Gas flow crossing the bubble $\left[\mathrm{m}^{2} / \mathrm{s}\right]$

$R_{b}$ : Bubble radius [m]

$r$ : Radial distance from the center of the bubble $[\mathrm{m}]$

$U$ : Superficial gas velocity $[\mathrm{m} / \mathrm{s}]$

$U_{0}$ : Superficial gas velocity far from the bubble $[\mathrm{m} / \mathrm{s}]$

$U_{b}$ : Gas velocity crossing the bubble $[\mathrm{m} / \mathrm{s}]$

$u$ : Gas velocity $[\mathrm{m} / \mathrm{s}]$

ũ: Relative gas velocity $[\mathrm{m} / \mathrm{s}$ ]

$v$ : Particle velocity $[\mathrm{m} / \mathrm{s}]$

$\beta$ : Function that defines the drag force $\left[\mathrm{kg} /\left(\mathrm{s} \mathrm{m}^{3}\right)\right]$

$\varepsilon$ : Voidage [ ]

$\varepsilon_{b}$ : Mean voidage at the bubble interior [

$\varepsilon_{b c}$ : Voidage at the bubble contour [ ]

$\varepsilon_{m f}$ : Voidage at minimum fluidization conditions [ ]

$\theta$ : Angle formed with the horizontal [rad]

$\mu$ : Gas viscosity [Pa s]

$\rho_{p}$ : Particle density $\left[\frac{\mathrm{kg}}{\mathrm{m}^{3}}\right]$

$\phi$ : Particle sphericity $(=1)[\quad]$

() : Inside surface of the bubble

$\mathrm{C}_{+}$: Outside surface of the bubble 